\title{
Eigenspine: Eigenvector Analysis of Spinal Deformities in Idiopathic Scoliosis
}

\author{
Daniel Forsberg, Claes Lundström, Mats Andersson and Hans Knutsson
}

\section{Linköping University Post Print}

\section{Tweet}

N.B.: When citing this work, cite the original article.

Original Publication:

Daniel Forsberg, Claes Lundström, Mats Andersson and Hans Knutsson, Eigenspine: Eigenvector Analysis of Spinal Deformities in Idiopathic Scoliosis, 2014, Computational Methods and Clinical Applications for Spine Imaging: Proceedings of the Workshop held at the 16th International Conference on Medical Image Computing and Computer Assisted Intervention, September 22-26, 2013, Nagoya, Japan, (), , 123-134.

http://dx.doi.org/10.1007/978-3-319-07269-2_11

Postprint available at: Linköping University Electronic Press

http://urn.kb.se/resolve?urn=urn:nbn:se:liu:diva-108975 


\title{
Eigenspine: Eigenvector Analysis of Spinal Deformities in Idiopathic Scoliosis
}

\author{
Daniel Forsberg ${ }^{1,2,3}$, Claes Lundström ${ }^{2,3}$, Mats Andersson ${ }^{1,2}$ and Hans Knutsson ${ }^{1,2}$
}

\begin{abstract}
In this paper, we propose the concept of eigenspine, a data analysis scheme useful for quantifying the linear correlation between different measures relevant for describing spinal deformities associated with spinal diseases, such as idiopathic scoliosis. The proposed concept builds upon the use of principal component analysis (PCA) and canonical correlation analysis (CCA), where PCA is used to reduce the number of dimensions in the measurement space, thereby providing a regularization of the measurements, and where CCA is used to determine the linear dependence between pair-wise combinations of the different measures. To demonstrate the usefulness of the eigenspine concept, the measures describing position and rotation of the lumbar and the thoracic vertebrae of 22 patients suffering from idiopathic scoliosis were analyzed. The analysis showed that the strongest linear relationship is found between the anterior-posterior displacement and the sagittal rotation of the vertebrae, and that a somewhat weaker but still strong correlation is found between the lateral displacement and the frontal rotation of the vertebrae. These results are well in-line with the general understanding of idiopathic scoliosis. Noteworthy though is that the obtained results from the analysis further proposes axial vertebral rotation as a differentiating measure when characterizing idiopathic scoliosis. Apart from analyzing pair-wise linear correlations between different measures, the method is believed to be suitable for finding a maximally descriptive low-dimensional combination of measures describing spinal deformities in idiopathic scoliosis.
\end{abstract}

\footnotetext{
${ }^{1}$ Department of Biomedical Engineering, Linköping University, Sweden

${ }^{2}$ Center for Medical Image Science and Visualization, Linköping University, Sweden

${ }^{3}$ Sectra, Linköping, Sweden

e-mail: $\{$ da-for, cl-lun\} @sectra.se, \{matsa, knutte\}@imt.liu.se
} 


\section{Introduction}

Idiopathic scoliosis is a disease affecting the spine by causing an excessive lateral curvature, as observed in the frontal plane, and is estimated to have a prevalence rate of 2 - 3\% for the age group 10 - 16 years old $[13,18]$. The disease is typically categorized according to curvature type $(\mathrm{C}$ - or $\mathrm{S}$-like), location of the primary curvature (thoracic, lumbar or thoracolumbar) and age of onset (infantile, juvenile, adolescent or adult). The choice of treatment, i.e. bracing or surgery, is dependent on a number of factors, which include age of onset, gender, skeletal maturity, the Cobb angle and the estimated progression rate. Especially the Cobb angle plays an important role in deciding which treatment to use. The Cobb angle is defined as the angle between two lines drawn parallel to the superior endplate of the superior end vertebra and parallel to the inferior endplate of the inferior end vertebra as observed in an anterior-posterior radiograph [1]. However, although an established measure, the Cobb angle measures a 2D projection of what is actually a 3D deformity, and, therefore, the relevance of using the Cobb angle alone for assessment of the spinal deformity of a scoliotic curvature can be questioned. To this end, a number of other methods for assessing spinal deformity have been developed, including both manual and computerized methods. Examples include methods for axial vertebral rotation measurements $[11,17]$, based on either 2D or 3D data, and methods for estimating both the position and the rotation of each vertebra $[2,5,16]$.

Apart from developing methods that can provide a more accurate 3D description of the spinal deformity of a scoliotic curvature, there is also a need to analyze how different measures describing spinal deformities relate to each other and to the clinical outcome $[4,6,15]$. This is important in order to classify various sub-types of idiopathic scoliosis and to determine if different treatments are suitable for the different sub-types of idiopathic scoliosis. Examples of this kind of work are found in $[3,7,14]$, where they apply clustering algorithms to the measures derived from the EOS system [2], in order to identify various sub-types of idiopathic scoliosis. However, there has been limited amount of work performed, aimed at analyzing the relation between different measures relevant for assessing spinal deformities. To this end, we present the concept of eigenspine, a data analysis scheme for analyzing the linear correlation between different measures relevant for describing spinal deformities.

\section{Eigenspine}

The proposed data analysis scheme is based on a combination of principal component analysis (PCA) and canonical correlation analysis (CCA), where PCA is used to reduce the number of dimensions in the measurement space, i.e. a regularization, and CCA is used to determine the linear dependence between pair-wise combinations of the different measures. Although, in this work, the proposed scheme is primarily employed to analyze the linear dependence between various measures, the 
long-term goal of the analysis is to determine which measures, or combination of measures, that are significant for describing and assessing a scoliotic curvature and, thus, providing an approach for creating a classification scheme similar to the ones that are typically used, e.g. King and Lenke [10, 12], but in this case relying on a $3 \mathrm{D}$ description of the deformity, instead of merely using $2 \mathrm{D}$ projections of a $3 \mathrm{D}$ deformity.

\subsection{PCA and CCA}

PCA and CCA are two standard techniques for exploring data and is typically applied in unsupervised learning. For the sake of completeness, we will briefly introduce the two methods. Let $\mathrm{X}$ denote a data matrix

$$
\mathrm{X}=\left[\begin{array}{llll}
\mathbf{x}_{1} & \mathbf{x}_{2} & \cdots & \mathbf{x}_{n}
\end{array}\right]
$$

where

$$
\mathbf{x}_{i}=\left[\begin{array}{llll}
x_{1} & x_{2} & \cdots & x_{p}
\end{array}\right]^{T},
$$

i.e. $\mathrm{X}$ contains $n$ measurements of $p$ variables. Compute the covariance matrix $\mathbf{C}_{X}$ as

$$
\mathbf{C}_{X} \approx \frac{1}{n-1}(\mathrm{X}-\overline{\mathrm{X}})(\mathrm{X}-\overline{\mathrm{X}})^{T}
$$

Define similarly a data matrix $\mathrm{Y}$.

For PCA, a linear transform $\mathrm{W}$ is estimated such that the variance of the components of $\mathrm{Z}=\mathrm{W}^{T} \mathrm{X}$ is maximized under the constraint that the components $\mathbf{w}_{i}$ of $\mathrm{W}$ are orthogonal, i.e. the components of $\mathrm{Z}$ are uncorrelated and $\mathbf{C}_{Z}=\mathrm{W}^{T} \mathbf{C}_{X} \mathrm{~W}$ is diagonal. In CCA, two linear transforms, $\mathrm{W}_{X}$ and $\mathrm{W}_{Y}$, are estimated such that the correlation $\rho_{i}$ between the reduced variables (canonical variates) of $\mathrm{W}_{X, i}^{T} \mathrm{X}$ and $\mathrm{W}_{Y, i}^{T} \mathrm{Y}$, have been maximized and that the different components of $\mathrm{W}_{X, i}^{T} \mathrm{X}$ and $\mathrm{W}_{Y, i}^{T} \mathrm{Y}$ are uncorrelated with respect to each other. Note that for CCA, the data matrices $X$ and $\mathrm{Y}$ are not required to have the same number of variables, therefore the number of canonical variates will correspond to the smallest number of variables provided by either $\mathrm{X}$ or $\mathrm{Y}$. Estimating the linear transforms $\mathrm{W}$ in PCA, and $\mathrm{W}_{X}$ and $\mathrm{W}_{Y}$ in $\mathrm{CCA}$ are done solving an eigenvector problem, hence, the term eigenspine.

An interesting aspect of CCA is its relation with mutual information (MI). As shown by [9], the mutual information between $\mathrm{X}$ and $\mathrm{Y}$ can be estimated as the sum of the mutual information of the reduced variables, given that their statistical dependence is limited to correlation. For normally distributed variables, this relation is given as

$$
\mathrm{MI}(\mathrm{X}, \mathrm{Y})=\frac{1}{2} \sum_{i} \log _{2}\left(\frac{1}{\left(1-\rho_{i}^{2}\right)}\right)
$$

This follows from considering a continuous random variable $\mathbf{x}$ with the differential entropy defined as 


$$
h(\mathbf{x})=-\int_{\mathbb{R}^{N}} p(\mathbf{x}) \log _{2}(p(\mathbf{x})) d \mathbf{x},
$$

where $p(\mathbf{x})$ is the probability density function of $\mathbf{x}$. Consider similarly a continuous random variable $\mathbf{y}$, then it can be shown that

$$
\operatorname{MI}(\mathbf{x}, \mathbf{y})=h(\mathbf{x})+h(\mathbf{y})-h(\mathbf{x}, \mathbf{y})=\int_{\mathbb{R}^{N}} \int_{\mathbb{R}^{M}} p(\mathbf{x}, \mathbf{y}) \log _{2}\left(\frac{p(\mathbf{x}, \mathbf{y})}{p(\mathbf{x}) p(\mathbf{y})}\right) d \mathbf{x} d \mathbf{y} .
$$

Further, consider a Gaussian distributed variable $\mathbf{z}$, for which the differential entropy is given as

$$
h(\mathbf{z})=\frac{1}{2} \log _{2}\left((2 \pi e)^{N}|\mathbf{C}|\right),
$$

where $\mathbf{C}$ is the covariance matrix of $\mathbf{z}$. In the case of two $N$-dimensional variables, then (6) becomes

$$
\operatorname{MI}(\mathbf{x}, \mathbf{y})=\frac{1}{2} \log _{2}\left(\frac{\left|\mathbf{C}_{x x}\right|\left|\mathbf{C}_{y y}\right|}{\mathbf{C}}\right)
$$

where

$$
\mathbf{C}=\left[\begin{array}{ll}
\mathbf{C}_{x x} & \mathbf{C}_{x y} \\
\mathbf{C}_{y x} & \mathbf{C}_{y y}
\end{array}\right]
$$

For two one-dimensional Gaussian distributed variables, (8) reduces to

$$
\operatorname{MI}(x, y)=\frac{1}{2} \log _{2}\left(\frac{\sigma_{x}^{2} \sigma_{y}^{2}}{\sigma_{x}^{2} \sigma_{y}^{2}-\sigma_{x y}^{2}}\right)=\frac{1}{2} \log _{2}\left(\frac{1}{1-\rho_{x y}^{2}}\right),
$$

where $\sigma_{x}^{2}$ and $\sigma_{y}^{2}$ are the variances of $x$ and $y, \sigma_{x y}^{2}$ is the covariance of $x$ and $y$ and $\rho_{x y}$ is the correlation between $x$ and $y$. Given that information is additive, for statistically independent variables, and that the canonical variates are uncorrelated, i.e. $\mathrm{W}_{X, i}^{T} \mathrm{X}$ and $\mathrm{W}_{Y, i}^{T} \mathrm{Y}$, hence, the mutual information between $\mathbf{X}$ and $\mathbf{Y}$ is the sum of the mutual information between the variates.

Note that using the log-function with the base 2 provides an MI measure defined in bits. This measure will be employed in the subsequent analysis for quantifying the dependence between different measures.

\section{Experiments}

To demonstrate the use of the data analysis scheme, measurements of the position and the orientation of the vertebrae for a number of patients were analyzed to determine which of these measures that have the strongest linear dependence. 


\subsection{Image Data}

Image data from 22 patients (19 female and four male) were retrospectively gathered and extracted from the local picture archiving and communications system. The only criteria for inclusion was that the patient suffered from idiopathic scoliosis and that the CT data had a resolution higher than $1 \times 1 \times 1 \mathrm{~mm}^{3}$. The data sets depicted all lumbar and thoracic vertebrae, i.e. 17 vertebrae per patient. The requirement on the resolution was needed in order to be able to distinguish adjacent vertebrae in the subsequently applied method for obtaining the position and rotation of each vertebra. The patients had an average age of $16.0 \pm 3.1$ years at the time of their respective examinations and an average Cobb angle of $60.4^{\circ} \pm 9.6$ (standing position). Most patients were classified has having a scoliosis of Lenke type $3 \mathrm{C}$ or $4 \mathrm{C}$.

The images were captured as a part of the standard routine for pre-operational planning and they were anonymized before being exported by clinical staff. Note that for patients of similar age as included in this retrospective study, it is often questionable whether a CT scan is appropriate or not, due to the exposure to radiation. However, at the local hospital there is a protocol in place for acquiring low-dose CT examinations with maintained image quality, targeted towards examinations of the spine. With the use of this protocol, the radiation dose is approximately $0.4 \mathrm{mSv}$. More on this can be found in [8].

\subsection{Curvature Measures}

Each data set was processed with the method presented in [5], which is based on the following steps; extraction of the spinal canal centerline, disc detection, vertebra centerpoint estimation and vertebra rotation estimation. A graphical overview of the method is provided in Fig. 1. In [5], the method was shown to have a variability, when compared with manual measurements, that was on par with inter-observer variability for measuring the axial vertebral rotation. This was supported by BlandAltman plots and high values of the intraclass correlation coefficient, thus, showing the method can be used as a replacement for manual measurements.

The method estimates, for each vertebra, the position $[x, y, z]$ and the rotation matrix $\mathrm{R}$, from which the rotation angles $\left[\theta_{X}, \theta_{Y}, \theta_{Z}\right]$ can be derived. The rotation angles were computed as the Euler angles (using a fixed world frame) of the rotation matrix $\mathrm{R}$. Note the order of the rotational angles, $\mathrm{R}=\mathrm{R}_{Z}\left(\theta_{Z}\right) \mathrm{R}_{Y}\left(\theta_{Y}\right) \mathrm{R}_{X}\left(\theta_{X}\right)$. $\theta_{Z}$ corresponds to axial vertebral rotation, $\theta_{Y}$ to frontal rotation and $\theta_{X}$ to sagittal rotation. The standard DICOM patient coordinate system was employed to define the orientation of $[x, y, z]$, i.e. $x$ increases from right to left, $y$ from anterior to posterior and $z$ from inferior to superior. 

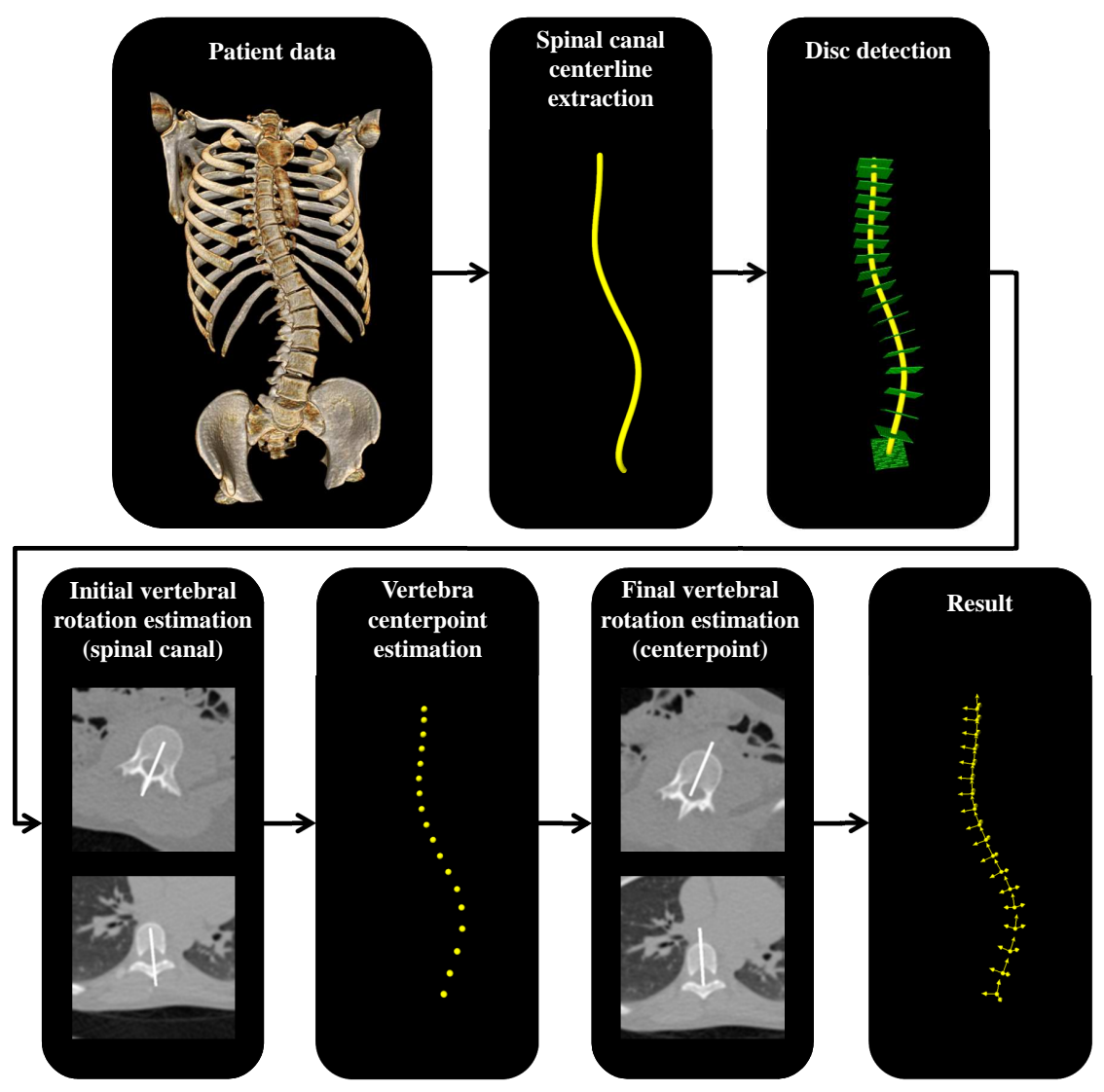

Fig. 1 An overview of the method for automatic measurements of the pose of each vertebra in the spine.

\subsection{Curvature Analysis}

In the data analysis, the curves of each measure, apart from the $z$-coordinates, for the entire spine were analyzed. The estimated $z$-coordinates were neglected and the vertebrae indexes were set to define the dimensions of the measurements per curve, i.e. each measure was embedded into a 17 dimensional space. Note that the $z$-coordinates were neglected, since the analysis is performed considering all measurements for a specific measure and patient simultaneously. Furthermore, the $x$ and $y$-coordinates of all vertebrae where translated in order to have the common starting point $(0,0)$ for all L5 vertebrae. Each curve ensemble was then processed with PCA to find its principal components. After the PCA, CCA was applied on the measures projected onto the subspace spanned by the largest PCA eigenvectors of each measure. The CCA was applied to analyze the dependence between all pairwise combinations of the different measures. 
A reasonable question at this point is why CCA is not applied directly on the estimated measures. The reason for this is two-fold. First, due to the large number of variables (17 vertebrae) compared to the low number of observations (22 patients), which can cause singularities in the computations of the CCA, a dimension reduction was called for. Second, using CCA directly is likely to generate an overfitting, i.e. it can find correlations related more to noise in the signal than to the relevant variations in the signal, hence, smoothing or a regularization was called for. Both of these requirements can be met by performing a PCA and projecting the signal onto the subspace spanned by the eigenvectors.

\section{Results}

In the conducted experiments, PCA was applied to each estimated measure, apart from the $z$-coordinates, over all patients, followed by a CCA on each pair-wise combination of the measures. From the estimated eigenvalues, the eigenvectors, corresponding to at least $99 \%$ of the variance in the data, were extracted. This meant for instance that the variance in $x$-coordinates could be reduced to four eigenvectors, whereas the curves of $\theta_{X}$ required six eigenvectors. Figure 2 depicts the measurements over all measures and patients, and Fig. 3 the extracted eigenvectors for each measure. Figure 4 depicts the reconstruction error between the original curves and the curves projected onto the subspace of the extracted eigenvectors.

The CCA was applied onto every pair-wise combination of the measures, where the measures were projected onto the subspace spanned by the extracted eigenvectors from the PCA. This was done to find dependencies between the different measures. Table 1 provides the obtained canonical correlations of all pair-wise CCAs along with corresponding MI estimates. Note the difference in number of canonical correlation coefficients between the different pair-wise comparisons, which is due to the different number of extracted eigenvectors per measure.

The results show that the strongest linear dependence exists between the $y$ coordinates and $\theta_{X}$. A somewhat weaker but still obvious dependence is found between the $x$-coordinates and $\theta_{Y}$. A second group of linear relationships, however, substantially weaker than the first two, is found between $\theta_{Y}$ and $\theta_{Z}$, and between $x$ and $\theta_{Z}$. Or, as expressed in anatomical terms, the anterior-posterior displacement of the vertebral body is highly correlated with the sagittal rotation of the same. In addition, lateral displacement and frontal rotation of the vertebrae are highly correlated. Substantially weaker but still obvious correlations are also found between axial vertebral rotation and both frontal rotation and lateral displacement. 
Table 1 Canonical correlations and MI as obtained from the pair-wise CCA, sorted according to estimated MI. Here $\rho_{i}$ denotes the correlation between the canonical variates, e.g. between $\mathrm{W}_{X, i}^{T} \mathrm{X}_{y}$ and $\mathrm{W}_{Y, i}^{T} \mathrm{Y}_{\theta_{X}}$ and where $\rho_{i} \geq \rho_{i+1}$. The MI-values are estimated in accordance with (4).

\begin{tabular}{lccccccr}
\hline & $\rho_{1}$ & $\rho_{2}$ & $\rho_{3}$ & $\rho_{4}$ & $\rho_{5}$ & $\rho_{6}$ & MI \\
\hline$y-\theta_{X}$ & 1.00 & 0.99 & 0.99 & 0.97 & & & 13.01 \\
$x-\theta_{Y}$ & 1.00 & 0.99 & 0.99 & 0.80 & & & 10.69 \\
$\theta_{Y}-\theta_{Z}$ & 0.99 & 0.96 & 0.92 & 0.81 & 0.39 & & 7.10 \\
$x-\theta_{Z}$ & 0.99 & 0.94 & 0.90 & 0.67 & & & 6.09 \\
$\theta_{X}-\theta_{Z}$ & 0.90 & 0.87 & 0.72 & 0.70 & 0.49 & 0.15 & 3.46 \\
$\theta_{X}-\theta_{Y}$ & 0.86 & 0.78 & 0.75 & 0.60 & 0.35 & & 2.67 \\
$y-\theta_{Z}$ & 0.88 & 0.70 & 0.53 & 0.36 & & & 1.88 \\
$x-\theta_{X}$ & 0.78 & 0.74 & 0.53 & 0.43 & & & 1.65 \\
$y-\theta_{Y}$ & 0.82 & 0.72 & 0.48 & 0.13 & & & 1.55 \\
$x-y$ & 0.71 & 0.49 & 0.45 & 0.16 & & & 0.90 \\
\hline
\end{tabular}
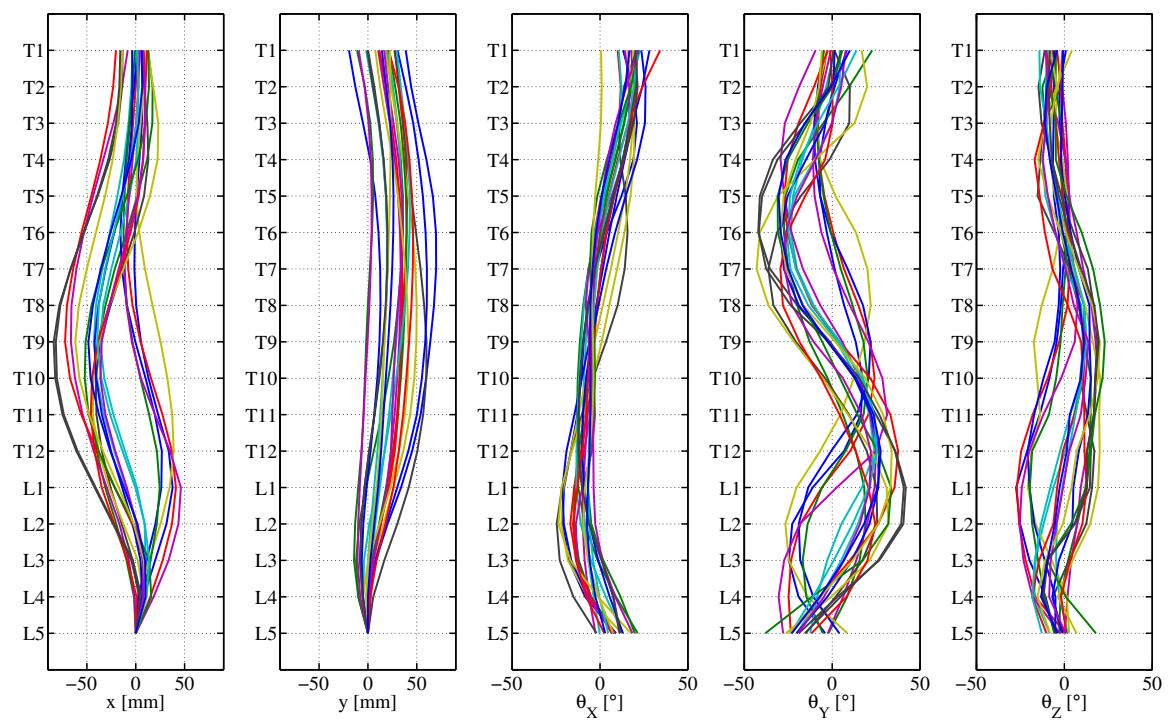

Fig. 2 All estimated measurements for all measures and all patients are depicted.

\section{Discussion}

This paper has presented the concept of eigenspine, a data analysis scheme for determining the relationship between different measures related to spinal deformity. The potential usage of the method has been exemplified by analyzing the dependence between different measures describing spinal deformities. The results of the combined PCA and CCA analysis show, for example, that the strongest linear dependencies are found between the anterior-posterior displacement and sagittal rotation of the vertebral body and between the lateral displacement and frontal rotation of 
Eigenspine: Eigenvector Analysis of Spinal Deformities in Idiopathic Scoliosis
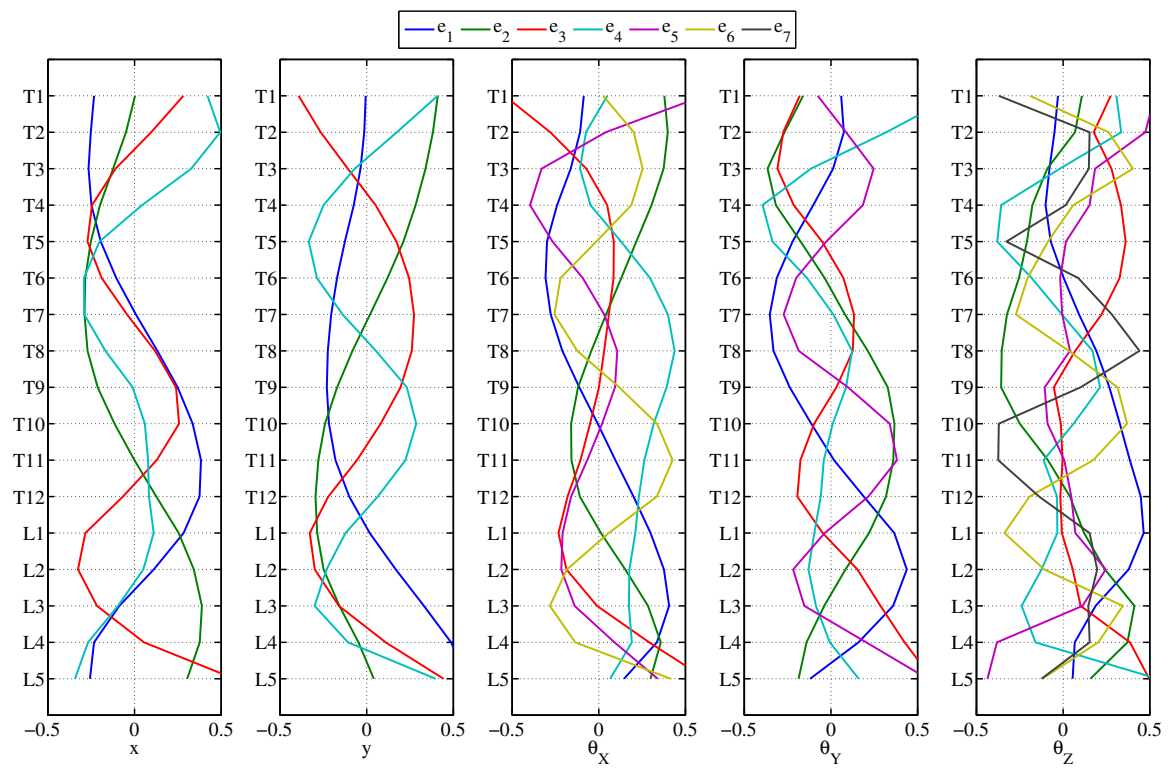

Fig. 3 Eigenvectors belonging to the largest eigenvalues as estimated from the PCA, accounting for at least $99 \%$ of the variance in the data.
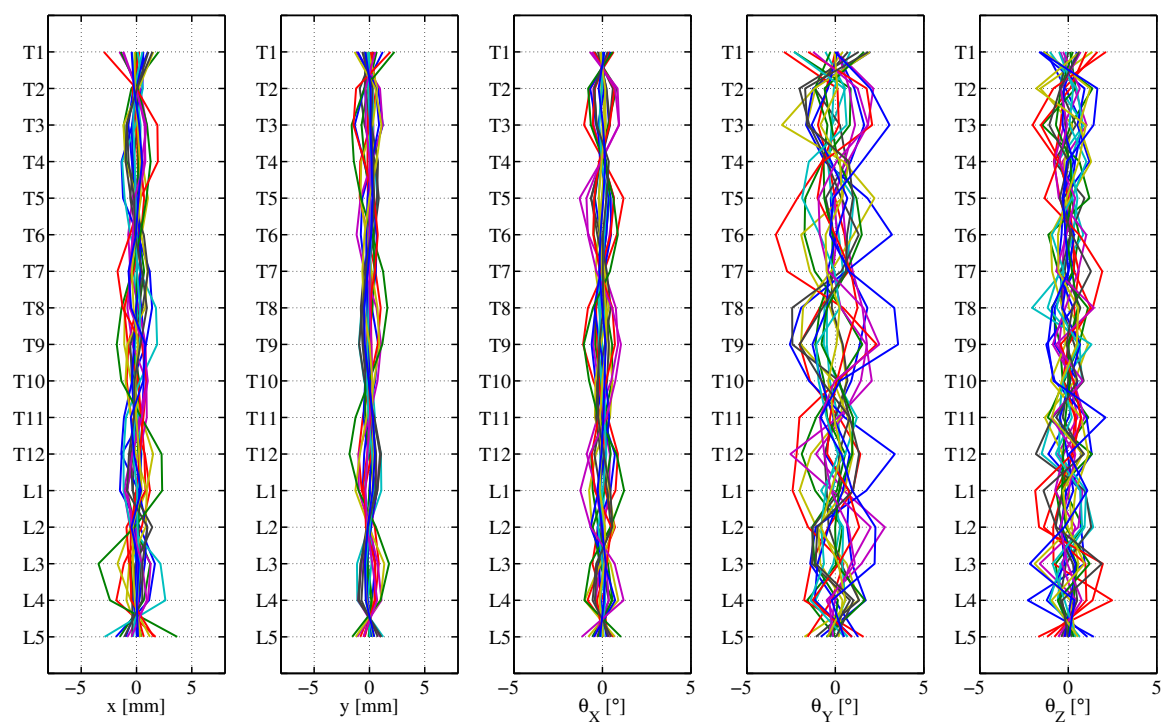

Fig. 4 Reconstruction error as given by the difference between the original curves and the curves projected onto the subspace of the eigenvectors accounting for $99 \%$ of the variance in the data.

the vertebrae. That there is a strong linear dependence between these measures is 
well in-line with what would be expected and what is previously known. However, more interesting conclusions can be drawn, once the different pair-wise linear dependencies are compared, since this analysis can indicate which measures that are the most relevant for describing a scoliotic curvature.

For instance, the fact that the strongest linear relationships exist between the pairwise measures $y-\theta_{X}$ and $x-\theta_{Y}$ indicates that to describe a scoliotic curvature it suffices to either measure the sagittal rotation $\theta_{X}$ and the frontal rotation $\theta_{Y}$ or the lateral and the anterior-posterior displacements $x$ and $y$. Given that there is some linear relationship between axial vertebral rotation $\theta_{Z}$ and both frontal rotation $\theta_{Y}$ and lateral displacement $x$, but that it is substantially weaker then the two primary linear correlations, a tentative hypothesis would be that axial vertebral rotation $\theta_{Z}$ is a differentiating factor when describing a scoliotic curvature. An understanding that adds support to the recent interest in quantifying the axial vertebral rotation when assessing idiopathic scoliosis. This further indicates that the classification systems by King and by Lenke [10,12], are insufficient to fully differentiate between different types of scoliosis, since the axial vertebral rotation is not included in their respective classification systems. However, given the limited number of included patients, further analysis including more patients is called for, before any conclusions can be made.

It is important to point out that the obtained quantification of the linear dependencies between all pair-wise combinations of measures via the computed MI, relies on the assumption of normal distributions for all included variables. An assumption that is questionable whether it holds, and, thus, needs further analysis.

Further, it can be noted that the data analysis scheme has been employed to measures based upon a method for estimating the pose of each vertebra as derived from CT data. However, the eigenspine concept is not limited to these measures or the used method, but could be readily applied to other measurements obtained with e.g. the EOS system. An analysis based upon the data employed in $[3,7,14]$ would be interesting to pursue in order to further quantify the relation between different measures, since the patient groups employed therein are rather large. Another interesting future aspect would be to include other measures, e.g. measures related to the deformation that the vertebral bodies undergo during the progression of the scoliotic curvature.

In this work, only the linear correlation between pair-wise combinations of measures have been analyzed. However, using CCA we believe it would be possible to extend this analysis further, but instead analyzing information content for any number of combined measures by employing autocorrelation. This could be useful to determine a maximally descriptive low-dimensional combination of measures describing spinal deformities in idiopathic scoliosis, and thereby providing means to better relate treatment and outcome of different types of idiopathic scoliosis, which would be a significant clinical outcome.

Acknowledgements The authors would like to thank L. Vavruch and H. Tropp at the Department of Clinical and Experimental Medicine, Linköping University, Sweden, for valuable input in discussions regarding idiopathic scoliosis and for assistance in collecting the used image data. This 
Eigenspine: Eigenvector Analysis of Spinal Deformities in Idiopathic Scoliosis

work was funded by the Swedish Research Council (grant 2007-4786) and the Swedish Foundation for Strategic Research (grant SM10-0022).

\section{References}

1. Cobb, R.: Outline for study of scoliosis. American Academy of Orthopaedic Surgeons, Instructional Course Lectures pp. 261-275 (1948)

2. Dubousset, J., Charpak, G., Dorion, I., Skalli, W., Lavaste, F., Deguise, J., Kalifa, G., Ferey, S.: A new 2D and 3D imaging approach to musculoskeletal physiology and pathology with lowdose radiation and the standing position: the EOS system. Bulletin de l'Academie nationale de medecine 189(2), 287 (2005)

3. Duong, L., Cheriet, F., Labelle, H.: Three-dimensional classification of spinal deformities using fuzzy clustering. Spine 31(8), 923-930 (2006)

4. Easwar, T., Hong, J., Yang, J., Suh, S., Modi, H.: Does lateral vertebral translation correspond to Cobb angle and relate in the same way to axial vertebral rotation and rib hump index? A radiographic analysis on idiopathic scoliosis. European Spine Journal 20(7), 1095-1105 (2011)

5. Forsberg, D., Lundström, C., Andersson, M., Vavruch, L., Tropp, H., Knutsson, H.: Fully automatic measurements of axial vertebral rotation for assessment of spinal deformity in idiopathic scoliosis. Physics in Medicine and Biology 58(6), 1775-1787 (2013)

6. Hong, J.Y., Suh, S.W., Easwar, T.R., Modi, H.N., Yang, J.H., Park, J.H.: Evaluation of the three-dimensional deformities in scoliosis surgery with computed tomography: Efficacy and relationship with clinical outcomes. Spine 36(19), E1259-E1265 (2011)

7. Kadoury, S., Labelle, H.: Classification of three-dimensional thoracic deformities in adolescent idiopathic scoliosis from a multivariate analysis. European Spine Journal 21(1), 40-49 (2012)

8. Kalra, M.K., Quick, P., Singh, S., Sandborg, M., Persson, A.: Whole spine CT for evaluation of scoliosis in children: feasibility of sub-millisievert scanning protocol. Acta Radiologica 54(2), 226-230 (2013)

9. Kay, J.: Feature discovery under contextual supervision using mutual information. In: Neural Networks, 1992. IJCNN., International Joint Conference on, vol. 4, pp. 79-84. IEEE (1992)

10. King, H.A., Moe, J.H., Bradford, D.S., Winter, R.B.: The selection of fusion levels in thoracic idiopathic scoliosis. J Bone Joint Surg Am 65(9), 1302-13 (1983)

11. Lam, G., Hill, D., Le, L., Raso, J., Lou, E.: Vertebral rotation measurement: a summary and comparison of common radiographic and CT methods. Scoliosis 3(1), 16 (2008)

12. Lenke, L.G., Betz, R.R., Harms, J., Bridwell, K.H., Clements, D.H., Lowe, T.G., Blanke, K.: Adolescent idiopathic scoliosis a new classification to determine extent of spinal arthrodesis. The Journal of Bone \& Joint Surgery 83(8), 1169-1181 (2001)

13. Reamy, B.V., Slakey, J.B.: Adolescent idiopathic scoliosis: review and current concepts. American Family Physician 64(1), 111-117 (2001)

14. Sangole, A.P., Aubin, C.E., Labelle, H., Stokes, I.A.F., Lenke, L.G., Jackson, R., Newton, P.: Three-dimensional classification of thoracic scoliotic curves. Spine 34(1), 91-99 (2008)

15. Schwab, F.J., Smith, V.A., Biserni, M., Gamez, L., Farcy, J.P.C., Pagala, M.: Adult scoliosis: a quantitative radiographic and clinical analysis. Spine 27(4), 387-392 (2002)

16. Vrtovec, T.: Modality-independent determination of vertebral position and rotation in 3D. In: Medical Imaging and Augmented Reality, Lecture Notes in Computer Science, vol. 5128, pp. 89-97. Springer (2008)

17. Vrtovec, T., Pernuš, F., Likar, B.: A review of methods for quantitative evaluation of axial vertebral rotation. European Spine Journal 18, 1079-1090 (2009)

18. Weinstein, S.L., Dolan, L.A., Cheng, J.C., Danielsson, A., Morcuende, J.A.: Adolescent idiopathic scoliosis. The Lancet 371(9623), 1527-1537 (2008) 